LPT 96-08

TK 9609

nucl-th/9604nnn

\title{
Double neutral pion photoproduction at threshold\#
}

\author{
V. Bernard ${ }^{a}$, N. Kaiser ${ }^{b}$, Ulf-G. Meißner ${ }^{c}$ \# $^{2}$ \\ ${ }^{a}$ Laboratoire de Physique Théorique, Institut de Physique \\ 3-5 rue de l'Université, F-67084 Strasbourg Cedex, France \\ and \\ Centre de Recherches Nucléaires, Physique Théorique \\ BP 28, F-67037 Strasbourg Cedex 2, France \\ email: bernard@crnhp4.in2p3.fr \\ ${ }^{b}$ Technische Universität München, Physik Department T39 \\ James-Franck-Straße, D-85747 Garching, Germany \\ email: nkaiser@physik.tu-muenchen.de \\ ${ }^{c}$ Universität Bonn, Institut für Theoretische Kernphysik \\ Nussallee 14-16, D-53115 Bonn, Germany \\ email: meissner@itkp.uni-bonn.de
}

\begin{abstract}
We consider the chiral expansion of the threshold amplitude for the reaction $\gamma p \rightarrow \pi^{0} \pi^{0} p$ to order $\mathcal{O}\left(M_{\pi}^{2}\right)$. We substantiate a claim that this photoproduction channel is significantly enhanced close to threshold due to pion loops. A precise measurement of the corresponding cross sections is called for which allows to test chiral perturbation theory.
\end{abstract}

October 9, 2018

${ }^{\# 1}$ Work supported in part by the Deutsche Forschungsgemeinschaft.

\#2 Address after Sept. 1, 1996: FZ Jülich, IKP (Theorie), D-52425 Jülich, Germany 
1. Single pion photo- and electroproduction off protons have been investigated extensively both from the experimental and the theoretical sides over the last years. Complementary information can be gained by the study of double pion production by real and virtual photons. In Ref. [1] we presented an exploratory study of double pion photo- and electroproduction close to threshold in the framework of heavy baryon chiral perturbation theory. For the threshold amplitudes, the first two terms in the chiral expansion of orders one and $M_{\pi}$, respectively, were given. The most prominent result of that investigation was the finding that the production cross section for final states with two neutral pions is considerably enhanced due to chiral (pion) loops. In this letter, we wish to sharpen the prediction for this effect by calculating all next-to-leading order terms of order $M_{\pi}^{2}$ in the threshold amplitude. At this order, one also has to include the effects of higher dimension operators. The coefficients of a subset of these have been determined recently from $\pi N$ scattering data in the study of the reaction $\pi N \rightarrow \pi \pi N$ [2]. Furthermore, one has to account for the Roper resonance which plays a very important role in models that attempt to describe the energy dependence over a large range of excitation energies [3]. Such studies are complementary to ours which is based on the low energy effective field theory of QCD and thus allows to investigate the strictures of the broken chiral symmetry for small external momenta, i.e. it is valid exclusively in the threshold region. The existing data of the two-pion photoproduction reactions from DAPHNE [4] and TAPS [5] are not yet conclusive for the threshold region. However, recently new precision data have been taken with TAPS [6] for the reaction $\gamma p \rightarrow \pi^{0} \pi^{0} p$ also close to threshold which will be analysed in the near future. An improved calculation is therefore called for to allow new tests of chiral perturbation theory in this particular low energy reaction.

2. First, we have to give some necessary definitions. The threshold matrix element for double neutral pion photoproduction off protons in the center of mass frame takes the form

$$
T\left(\gamma p \rightarrow \pi^{0} \pi^{0} p\right)_{\mathrm{cm}}^{\mathrm{thr}}=i \vec{\sigma} \cdot(\vec{\epsilon} \times \vec{k}) \mathcal{M}_{00},
$$

where $\vec{\sigma}, \vec{\epsilon}$ and $\vec{k}$ denote the spin of the nucleon, the photon polarization vector and the photon three-momentum (in the $\mathrm{cm}$ frame), in order, subject to the transversality constraint $\vec{\epsilon} \cdot \vec{k}=0$. The (complex) threshold amplitude $\mathcal{M}_{00}$ encodes the physics. Assuming the photoproduction amplitude to be constant in the threshold region and approximating the three-body phase space and photon flux by a simple analytic formulat? the unpolarized total cross section near threshold is

$$
\sigma_{\text {tot }}\left(E_{\gamma}\right)=\frac{M_{\pi}^{2}(1+\mu)}{64 \pi^{2}(1+2 \mu)^{11 / 2}}\left|\mathcal{M}_{00}\right|^{2} \cdot\left(E_{\gamma}-E_{\gamma}^{\mathrm{thr}}\right)^{2},
$$

with $E_{\gamma}$ the photon lab energy and $\mu=M_{\pi} / m=0.144$ the ratio of the (neutral) pion to the proton mass. The value of the threshold photon energy in the lab frame is $E_{\gamma}^{\text {thr }}=$ $2 M_{\pi^{0}}(1+\mu)=308.8 \mathrm{MeV}$.

3. To calculate the chiral expansion of the threshold amplitude $\mathcal{M}_{00}$, we make use of heavy baryon chiral perturbation theory [7, []. Our notation is identical to the one used

\footnotetext{
\#3 This approximation has been shown to work excellently in [1].
} 
in Ref.[1] and we refer to that reference for further details. We will extend the calculation of that paper by working out all terms of order $q^{4}$ in the Lagrangian, which amounts to tree graphs with insertion from the dimension one, two, three and four effective pionnucleon Lagrangian as well as pion loop graphs with at most one dimension two insertion. Since the quantities $\vec{\epsilon}$ and $\vec{k}$ count as small momenta, we therefore get all terms up-toand-including $\mathcal{O}\left(M_{\pi}^{2}\right)$ in the chiral expansion of $\mathcal{M}_{00}$.

Let us now consider the various contributions. The tree graphs (sequential photon absorption and pion emissions on the proton line) with the lowest order couplings together with the higher order magnetic moment terms are conventionally called the Born terms. The Born contribution to the threshold amplitude is easily evaluated (notice that for double neutral pion production the first non-vanishing Born term starts at order $M_{\pi}$ ),

$$
\mathcal{M}_{00}^{\text {Born }}=\frac{e g_{A}^{2} M_{\pi}}{2 m^{2} F_{\pi}^{2}}\left[1-\frac{M_{\pi}}{4 m}\left(1+3 \kappa_{p}\right)\right]=(4.9-1.2) \mathrm{GeV}^{-3}=3.7 \mathrm{GeV}^{-3}
$$

using $e^{2} / 4 \pi=1 / 137.036, F_{\pi}=92.4 \mathrm{MeV}, M_{\pi}=139.57 \mathrm{MeV}, m=938.27 \mathrm{MeV}, \kappa_{p}=$ 1.793 and $g_{A}=1.32$ as determined from the Goldberger-Treiman relation (with $g_{\pi N}=$ 13.3). Isospin breaking via the difference of the neutral and charged pion masses is negligible here (3\%-effect). The one-pion loop diagrams contributing at order $q^{3}$ are taken from [回],

$$
\mathcal{M}_{00}^{\mathrm{q}^{3}-\text { loop }}=\frac{3 e g_{A}^{2} M_{\pi}}{128 \pi F_{\pi}^{4}}\left\{2+\frac{\pi}{2}+i[2 \sqrt{3}-\ln (2+\sqrt{3})]\right\}=(26.9+16.2 i) \mathrm{GeV}^{-3},
$$

where the imaginary part is related to the rescattering type of diagrams which have a right-hand cut starting at the single pion production threshold, $s=\left(M_{\pi}+m\right)^{2}$, which is well below the two pion production threshold. Notice that the $\mathcal{O}\left(q^{3}\right)$ loop contribution Eq.(11) is much larger than the one from the Born terms, Eq.(3). This is, indeed, the large chiral loop effect which enhances the cross section for the photoproduction of two neutral pions off protons considerably. At this order, one could also have $1 / \mathrm{m}$ suppressed tree graph contributions proportional to the low-energy constants $c_{i}$. As shown in [1], these vanish due to the threshold selection rules or cancel pairwise. However, at one order higher, one has such contributions. In the notation of Ref. [2], the terms proportional to the low energy constants $c_{i}$ read

$$
\mathcal{M}_{00}^{c_{i}}=\frac{e M_{\pi}^{2}}{m^{2} F_{\pi}^{2}}\left[-2 c_{1}+\left(1+\kappa_{p}\right) c_{2}^{\prime}+\left(1+2 \kappa_{p}\right) c_{2}^{\prime \prime}-c_{3}\right]=18.4 \mathrm{GeV}^{-3},
$$

using the central values of the $c_{i}$ as determined in [2] from a fit to the subthreshold pionnucleon scattering amplitudes, the $\pi N \sigma$-term and some $\pi N$ P-wave scattering volumes $\left(c_{1}=-0.64, c_{2}^{\prime}=-5.63, c_{2}^{\prime \prime}=7.41, c_{3}=-3.90\right.$, all in $\left.\mathrm{GeV}^{-1}\right)$. In a microscopic picture, these tree terms $\sim c_{i}$ subsume all $\mathrm{s}^{-}, \mathrm{t}-$ and $\mathrm{u}-$-channel resonance excitations in the $\pi^{0} p$ scattering amplitude. Combining these with photon absorption on the proton line, one is lead to a contribution to $\gamma p \rightarrow \pi^{0} \pi^{0} p$, i.e. the expression Eq.(5) for double neutral pion production off protons. The anatomy of such a chiral coefficient is discussed in [9], 
see also [10]. We note that contributions from single $\pi^{0}$-photoproduction via resonance exchanges (see Ref. [13]) followed by $\pi^{0}$ emission off the proton line vanish to the order we are working here.

Next, we consider the pion loops at order $q^{4}$. Many of these are similar in structure to the ones evaluated in [2] for the reaction $\pi N \rightarrow \pi \pi N$. Furthermore, one can show that the sum of all one-loop diagrams with one insertion proprotional to $c_{i}(i=1,2,3)$ vanishes. Consequently, only the dimension two operators with the LEC $c_{4}$ (central value $c_{4}=2.25 \mathrm{GeV}^{-1}$ [2]) together with the anomalous magnetic moments of the proton $\left(\kappa_{p}\right)$ and the neutron $\left(\kappa_{n}=-1.913\right)$ and $1 / m$ suppressed vertices give a contribution to the $q^{4}$ one loop result

$$
\begin{aligned}
\mathcal{M}_{00}^{\mathrm{q}^{4}-\text { loop }}= & \frac{e M_{\pi}^{2}}{64 \pi^{2} m F_{\pi}^{4}}\left\{2\left[\kappa_{p}\left(11 g_{A}^{2}-1\right)+\kappa_{n}\left(3 g_{A}^{2}+1\right)+20 g_{A}^{2}-12 m c_{4}-4\right] \ln \frac{M_{\pi}}{\lambda}\right. \\
& -\left(29 g_{A}^{2}+24 m c_{4}+6\right) \frac{\pi^{2}}{16}+g_{A}^{2} \pi+3\left(4 g_{A}^{2}-4 m c_{4}-1\right) \sqrt{2} \ln (1+\sqrt{2}) \\
& -3\left(g_{A}^{2}+2 m c_{4}+\frac{1}{2}\right) \ln ^{2}(1+\sqrt{2})+g_{A}^{2}\left(13+11 \kappa_{p}+3 \kappa_{n}\right) \sqrt{3} \ln (2+\sqrt{3}) \\
& -\frac{3}{4} g_{A}^{2} \ln ^{2}(2+\sqrt{3})+7+24 m c_{4}+\left(g_{A}^{2}-1\right) \kappa_{n}-\frac{g_{A}^{2}}{3}\left(37+31 \kappa_{p}\right)+\kappa_{p} \\
& \left.-i \frac{\pi}{2} g_{A}^{2}\left[\left(9+11 \kappa_{p}+3 \kappa_{n}\right) \sqrt{3}+\frac{11}{2} \ln (2+\sqrt{3})\right]\right\} \\
& =(-10.3-17.6 i) \mathrm{GeV}^{-3}
\end{aligned}
$$

Here, $\lambda$ is the scale of dimensional regularization. In what follows, we set $\lambda=m$. The (spurious) scale dependence is rather mild, for $\lambda=m^{*}=1.44 \mathrm{GeV}$, the real part in Eq.(6) changes to $-13.3 \mathrm{GeV}^{-3}$. We note that in contrast to the $q^{3}$ calculation, the loops are no longer finite. Furthermore, the imaginary part at orders $q^{3}$ and $q^{4}$ are of the same size but of opposite sign. These first two terms in the chiral expansion of $\operatorname{Im} \mathcal{M}_{00}$ therefore do not give a reliable estimate of the imaginary part. To get a better handle on it, we make use of the Fermi-Watson final-state theorem. Unitarity relates the imaginary part Im $\mathcal{M}_{00}$ to the rescattering process $\gamma p \rightarrow \pi N \rightarrow \pi^{0} \pi^{0} p$ with intermediate $\pi N$ states on mass-shell. The resulting exact expression is:

$$
\begin{aligned}
\operatorname{Im} \mathcal{M}_{00} & =-\frac{3 M_{\pi}(2+3 \mu)(2+\mu)}{8(1+2 \mu)(1+\mu)}\left[\sqrt{2} D_{2}^{*} M_{1-}^{\pi^{+} n}+\left(2 D_{1}^{*}+D_{2}^{*}\right) M_{1-}^{\pi^{0} p}\right] \\
& =\frac{3 M_{\pi}(2+3 \mu)(2+\mu)}{8(1+2 \mu)(1+\mu)}\left[\mathcal{A}_{10}^{*}{ }_{p} M_{1-}^{(1 / 2)}-\frac{2}{15} \sqrt{10} \mathcal{A}_{32}^{*} M_{1-}^{(3 / 2)}\right] \simeq 7 \mathrm{GeV}^{-3}
\end{aligned}
$$

where the factor in front of the brackets is the ratio of the intermediate pion momentum squared to the photon momentum, $\left|\vec{q}_{\pi}\right|^{2} /|\vec{k}|$ (in the center-of-mass system). $D_{1,2}$ are the threshold amplitudes for the reaction $\pi N \rightarrow \pi \pi N$ [2] and the $M_{1-}$ are the pertinent single pion photoproduction $\mathrm{P}$-wave magnetic dipole amplitudes. In the second line of Eq.(7), the decomposition into channels with definite total isospin $1 / 2$ and $3 / 2$, respectively, of the intermediate $\pi N$ system is given. The threshold $\pi \pi N$ amplitudes have the (central) 
values [2]

$$
D_{1}=2.26 \mathrm{fm}^{3}, \quad D_{2}=-9.05 \mathrm{fm}^{3}, \quad \mathcal{A}_{10}=8.01 M_{\pi}^{-3}, \quad \mathcal{A}_{32}=2.53 M_{\pi}^{-3},
$$

and for the photoproduction magnetic multipoles we take the values from the analysis of Berends and Donnachie [11] at $E_{\gamma}=310 \ldots 320 \mathrm{MeV}$ (see also Ref.[12]),

$$
{ }_{p} M_{1-}^{(1 / 2)} \simeq 1 \cdot 10^{-3} M_{\pi}^{-1}, \quad M_{1-}^{(3 / 2)} \simeq-6 \cdot 10^{-3} M_{\pi}^{-1},
$$

which leads to the value of $\operatorname{Im} \mathcal{M}_{00}$ given in Eq.([). The imaginary parts of the (actually complex) $\pi \pi N$ threshold and pion photoproduction magnetic dipole amplitudes are negligible for our estimate in Eq.(7), since the respective $\pi N$-phases $\left(\delta_{11} \simeq 2^{\circ}, \delta_{31} \simeq-4^{\circ}\right)$ are tiny. Even though the imaginary part $\operatorname{Im} \mathcal{M}_{00}$ calculated to order $q^{4}$ is numerically not reliable, it serves as an important check on the evaluation of the loop integrals.

Finally, we have to account for tree level insertions from the dimension three and four local contact terms. As detailed in Ref.[1], we make use of the resonance saturation hypothesis to estimate these. As argued before, single $\Delta$ excitation (see Fig. $5 \mathrm{a}-\mathrm{h}$ in [1]) is to order $q^{4}$ subsumed in the tree terms $\sim c_{i}$, Eq.(5), so we are left with double $\Delta$ graphs like in Fig. 5i,j, $\mathrm{k}$ in [1]. To proceed, we generate the $\Delta \Delta \gamma$ coupling by minimal substitution in the free $\Delta$ Lagrangian and the unknown $\Delta \Delta \pi$ coupling with help of the spin-3/2 axial current. Using the coupling constant relation $g_{\pi \Delta \Delta}=4 g_{\pi N} / 5$ from the $\mathrm{SU}(4)$ quark model, we get

$$
\begin{aligned}
\mathcal{M}_{00}^{\Delta \Delta}= & \frac{e g_{A}^{2} M_{\pi}^{2}}{2 m m_{\Delta}^{2} F_{\pi}^{2}}\left\{\frac{4}{15} \sqrt{2} g_{1}(1+2 Y)(1-2 Z)+\frac{m_{\Delta}}{m-m_{\Delta}}+\frac{m^{2}}{3 m_{\Delta}^{2}}(1-2 Z)^{2}\right. \\
& \left.+\frac{m}{3 m_{\Delta}}(1-2 Z)(5+2 Z)+\frac{7}{4}-Z-Z^{2}\right\}=1.2 \mathrm{GeV}^{-3}
\end{aligned}
$$

where the first term related to the graphs Fig. 5i,k in [1] (the photon does not couple to two $\left.\Delta^{+}\right)$dominates. The off-shell parameters $Y$ and $Z$ have been determined in [13] $\left(g_{1}=5, Y=0.1, Z=-0.2\right)$. Inserting these, we find that the double $\Delta$ contribution is negligible. This agrees with the findings of Bolokhov et al. [14] for the reaction $\pi N \rightarrow$ $\pi \pi N$. We remark that such terms are exactly zero in the non-relativistic limit (static isobar model) [3]. Another possible contribution comes from loop diagrams where the photon couples to a virtual $K^{+}$meson and $\Sigma^{0}$ and $\Lambda$ hyperons appear in the intermediate state. The respective calculation in chiral $S U(3)$ gives after expanding in inverse powers of the kaon mass

$$
\mathcal{M}_{00}^{\mathrm{K}^{+}-\text {loop }}=\frac{e\left(D^{2}+3 F^{2}\right) M_{\pi}^{2}}{48 \pi F_{\pi}^{4} M_{K}}=1.4 \mathrm{GeV}^{-3}
$$

using $M_{K}=493.6 \mathrm{MeV}$ and $D=0.76, F=0.50$ for the axial vector coupling constants. As in single pion photoproduction [15] 113] the (frozen) $K^{+}$-loop effects are negligibly small.

The largest resonance contribution at order $M_{\pi}^{2}$ comes indeed from the Roper resonance $N^{*}(1440)$. To be specific, the pertinent Feynman diagrams involve the $N^{*} N \pi \pi$ S-wave 
vertex which is discussed in detail in Ref. [2]. To leading order, it is parametrized in terms of two coupling constants, denoted $c_{1}^{*}$ and $c_{2}^{*}$, respectively. We also need the $N^{*+} p \gamma$ coupling, $\kappa^{*}$, defined via the relativistic vertex $\left(e \kappa^{*} / 4 m\right) \bar{\Psi} \sigma_{\mu \nu} F^{\mu \nu} \Psi^{*}$. The transition magnetic moment $\kappa^{*}$ can be determined from the Roper radiative decay width,

$$
\Gamma\left(N^{*+} \rightarrow p \gamma\right)=\frac{e^{2} \kappa^{* 2} k_{\gamma}^{3}}{4 \pi m^{2}}=\frac{k_{\gamma}^{2} m}{\pi m^{*}}\left|A_{1 / 2}\right|^{2}=0.18 \mathrm{MeV}, \quad \kappa^{*}=-0.56
$$

using the value $A_{1 / 2}=-0.072 \mathrm{GeV}^{-1 / 2}$ from the latest PDG tables and $k_{\gamma}=\left(m^{* 2}-\right.$ $\left.m^{2}\right) / 2 m^{*}=414 \mathrm{MeV}$. The strong couplings constants $c_{1,2}^{*}$ are only known within some broad ranges. We therefore give an upper limit for the Roper contribution using the maximal value of $c_{1}^{*}+c_{2}^{*}=-4.9 \mathrm{GeV}^{-1}$ [2]

$$
\mathcal{M}_{00}^{N^{*}}=\frac{2 e M_{\pi}^{2} \kappa^{*}\left(c_{1}^{*}+c_{2}^{*}\right)}{m\left(m^{*}-m\right) F_{\pi}^{2}}<11.7 \mathrm{GeV}^{-3} .
$$

The value $\mathcal{M}_{00}^{N^{*}}=11.7 \mathrm{GeV}^{-3}$ in Eq.(13) also includes the possible enhancement factor $\left[1-4 M_{\pi}^{2} /\left(m^{*}-m\right)^{2}\right]^{-1}=1.45$ which occurs if the energy denominators are not chirally expanded.\# If one chooses e.g. the central values of the Roper couplings $c_{1}^{*}+c_{2}^{*}=-1.6$ $\mathrm{GeV}^{-1}$ as in 2] this contribution is strongly reduced to $\mathcal{M}_{00}^{N^{*}}=2.6 \mathrm{GeV}^{-3}$. Obviously, the Roper contribution has presently the largest theoretical uncertainty. Higher resonances play therefore no role for the threshold amplitude $\mathcal{M}_{00}$, i.e. their contribution is well within the theoretical uncertainty.

4. We are now in the position to calculate the total cross section for $\gamma p \rightarrow \pi^{0} \pi^{0} p$ in the threshold region. Consider first the real part of $\mathcal{M}_{00}$. We find that the leading contribution of order $M_{\pi}$ is at least a factor 1.5 larger than the first correction at order $M_{\pi}^{2}$, $\operatorname{Re} \mathcal{M}_{00}^{q^{3}}=31.8 \mathrm{GeV}^{-3}$ and for the maximal value of the Roper contribution, $\operatorname{Re} \mathcal{M}_{00}^{q^{4}}=$ $21.2 \mathrm{GeV}^{-3}$, in order. For the central Roper couplings of [2] the order $M_{\pi}^{2}$ correction is even further reduced to $\operatorname{Re} \mathcal{M}_{00}^{q^{4}}=12.1 \mathrm{GeV}^{-3}$, which shows that the chiral expansion is well-behaved, i.e. the next-to-leading order correction is smaller than the leading term. For the maximal $N^{*}(1440)$ contribution together with the empirical imaginary part, we have

$$
\mathcal{M}_{00} \simeq(53+7 i) \mathrm{GeV}^{-3},
$$

which shows that the imaginary part is not important for the total cross section, i.e. it amounts to a 2\%-effect. Altogether, the predicted near threshold cross section is then

$$
\sigma_{\text {tot }}\left(E_{\gamma}\right) \leq 0.91 \mathrm{nb}\left(\frac{E_{\gamma}-E_{\gamma}^{\text {thr }}}{10 \mathrm{MeV}}\right)^{2}, \quad E_{\gamma}^{\text {thr }}=308.8 \mathrm{MeV}
$$

For comparison, at order $q^{3}$, the prefactor in Eq.(15) is $0.41 \mathrm{nb}$. We stress that the $0.91 \mathrm{nb}$ given in Eq.(15) are the upper limit. For the central Roper couplings in [2] which lead to

\footnotetext{
${ }^{\# 4}$ We are grateful to Eulogio Oset for pointing this out.

${ }^{\# 5}$ We use the neutral pion mass in the phase space factor as discussed in [1].
} 
a very good description of the $\pi \pi N$ threshold amplitude $D_{2}$, this number is reduced to $0.63 \mathrm{nb}$.

In summary, we have evaluated all next-to-leading order corrections to the threshold amplitude for the reaction $\gamma p \rightarrow \pi^{0} \pi^{0} p$. The dominant contribution still comes from the chiral loops at leading (non-vanishing) order, $q^{3}$. The higher order $q^{4}$ corrections are in total not very large and increase the cross section by about a factor 1.6 to 2.2 with the uncertainty mainly related to the size of Roper resonance contribution. In order to test the prediction Eq.(15) a precise measurement of these near threshold cross sections is now called for.

\section{Acknowledgements}

One of us (UGM) thanks the Institute for Nuclear Theory at the University of Washington for its hospitality and the Department of Energy for partial support during the completion of this work.

\section{References}

[1] V. Bernard, N. Kaiser, Ulf-G. Meißner and A. Schmidt, Nucl. Phys. A580 (1994) 475

[2] V. Bernard, N. Kaiser and Ulf-G. Meißner, Nucl. Phys. B475 (1995) 147

[3] J.A. Gómez Tejedor and E. Oset, Nucl. Phys. A571 (1994) 667;

"Double pion photoproduction on the nucleon: Study of the isospin channels", Nucl. Phys. A (1996) in press

[4] A. Braghieri et al., Phys. Lett. B363 (1995) 46

[5] F. Härter, H. Ströher, Th. Walcher et al., to be published

[6] H. Ströher, private communication

[7] E. Jenkins and A.V. Manohar, Phys. Lett. B255 (1991) 558

[8] V. Bernard, N. Kaiser, J. Kambor and Ulf-G. Meißner, Nucl. Phys. B388 (1992) 315

[9] Ulf-G. Meißner, in "Chiral Dynamics: Theory and Experiment", A.M. Bernstein and B.R. Holstein (eds.), Springer, Heidelberg, 1995

[10] V. Bernard, N. Kaiser and Ulf-G. Meißner, Int. J. Mod. Phys. E4 (1995) 193

[11] F.A. Berends and A. Donnachie, Nucl. Phys. B84 (1975) 342

[12] W. Pfeil and D. Schwela, Nucl. Phys. B45 (1972) 379 
[13] V. Bernard, N. Kaiser and Ulf-G. Meißner, "Chiral symmetry and the reaction $\gamma p \rightarrow$ $\pi^{0} p^{\prime \prime}$, Phys. Lett. B (1996) in press

[14] A.A. Bolokhov et al., Nucl. Phys. A592 (1995) 413

[15] S. Steininger and Ulf-G. Meißner, "Kaon loops in pion photoproduction: Facts and fancy", Bonn Univ. preprint TK 9604 nucl-th/9601048 